\title{
Total Synthesis of (+)-Fusarisetin A: A Biomimetic Approach
}

Jun Yin, Shuanhu Gao*

Shanghai Key Laboratory of Green Chemistry and Chemical Processes, Department of Chemistry, East China Normal University, 3663N Zhongshan Road, Shanghai 200062, P. R. of China

Fax +86(21)62604784; E-mail: shgao@chem.ecnu.edu.cn

Received: 26.08.2013; Accepted after revision: 17.09.2013

\begin{abstract}
This article outlines our recent efforts to synthesize $(+)$-fusarisetin A, a naturally occurring 3-acyltetramic acid, by a route based on a hypothetical biosynthesis. Our research suggests that the biosynthesis of (+)-fusarisetin A might involve the aerobic oxidation of equisetin, possibly mediated by metal oxidants or by photochemically produced reactive oxygen species.
\end{abstract}

Key words: radical reactions, total synthesis, biosynthesis, heterocycles, polycycles

Natural products have proven to be an indispensable source of human therapeutic agents. ${ }^{1}$ Many significant drugs are based on natural products or compounds derived from natural products, such as penicillin (antibiotic), quinine and artemisinin (antimalarials), lovastatin (lipid-control agent), and taxol and doxorubicin (anticancer drugs). ${ }^{2}$ Historically, these drugs have revolutionized human life by improving health standards and lengthening life expectancy. These natural therapeutics have also served as driving forces for the exploration of natural product synthesis, the growth of which not only promotes the development of new synthetic methodologies, but also provides a platform for further studies on medicinal chemistry and chem-

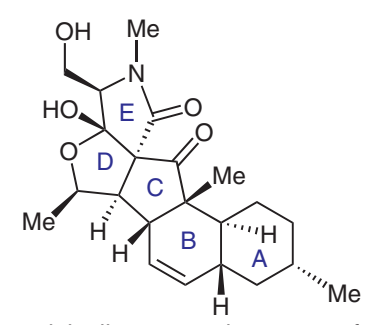

originally proposed structure of fusarisetin $\mathrm{A}$

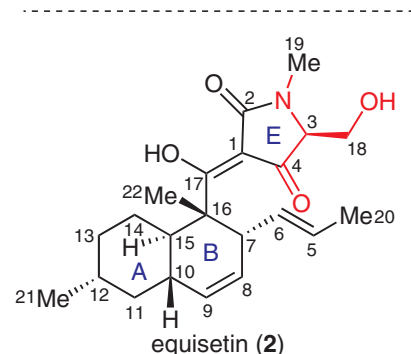

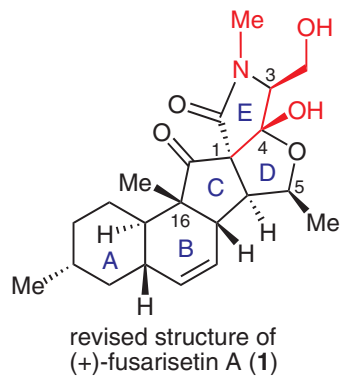

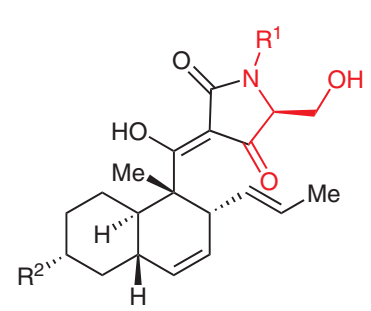

$\mathrm{R}^{1}=\mathrm{H}, \mathrm{R}^{2}=\mathrm{Me}$, trichosetin (3)

$\mathrm{R}^{1}=\mathrm{Me}, \mathrm{R}^{2}=\mathrm{CH}_{2} \mathrm{OH}$, ophiosetin (4)
Figure 1 Structures of (+)-fusarisetin A and biogenetically related natural products

SYNLETT 2014, 25, 0001-0007

Advanced online publication: 05.11.2013

DOI: 10.1055/s-0033-1340153; Art ID: ST-2013-P0821-SP

(C) Georg Thieme Verlag Stuttgart · New York

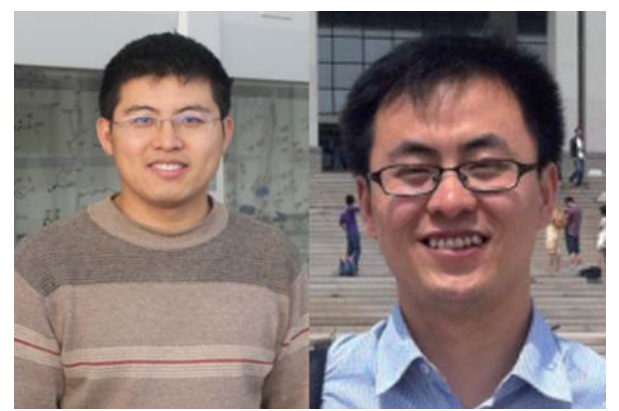

Jun Yin (left) was born in Shandong Province, China, in 1977. He received his bachelor's degree in 2000 and his master's degree in organic chemistry in 2003, studying under Professor Jianwu Wang at Shandong University. From 2003 to 2005, he worked at WuXi AppTec. (Shanghai) as a chemist. From 2006 to 2011, he worked at Roche R\&D Center (China) Ltd. as a medicinal chemist. In 2011, he joined Professor Shuanhu Gao's group to pursue doctoral studies at the Department of Chemistry of East China Normal University. His research projects focus on total synthesis of bioactive natural products.

Shuanhu Gao (right) was born in Ningxia Province, China, in 1979. He received his B.S. degree from Lanzhou University in 2001. In 2006 he obtained his Ph.D. from Lanzhou University under the direction of Professor Yongqiang Tu. From 2007 to 2010, he was a postdoctoral fellow in Professor Chuo Chen's group at the UT Southwestern Medical Center at Dallas. He began his independent career as a professor in the Department of Chemistry at East China Normal University in October 2010. His current research interests focus primarily on the synthesis of complex natural products and on medicinal chemistry.

ical biology. Therefore, natural product synthesis is an important aspect of both academic and pharmaceutical chemistry.

The goal of natural product synthesis has been to provide the target molecule efficiently and in adequate amounts; ${ }^{3}$ this requires practicing chemists to pay attention to such issues as chemoselectivity, ${ }^{4}$ atom economy, ${ }^{5 \mathrm{a}, \mathrm{b}}$ step economy, ${ }^{5 c-e}$ and redox economy. ${ }^{5 f, g}$ In this SynPact article, we wish to outline our recent efforts to achieve an efficient synthesis of (+)-fusarisetin A, a promising anticancer agent, based on a hypothetical biosynthesis of the molecule. We believe that our scalable synthesis will permit structure-activity relationship studies and facilitate further studies on the medicinal chemistry of this molecule.

Tetramic acid based natural products ${ }^{6}$ typically contain a pyrrolidine-2,4-dione moiety, which normally exists as a mixture of keto/enol tautomers (Scheme 1; I and II). Most tetramic acids carry acyl substituents in the $\mathrm{C}-3$ position and can therefore generally form four detectable tauto- 

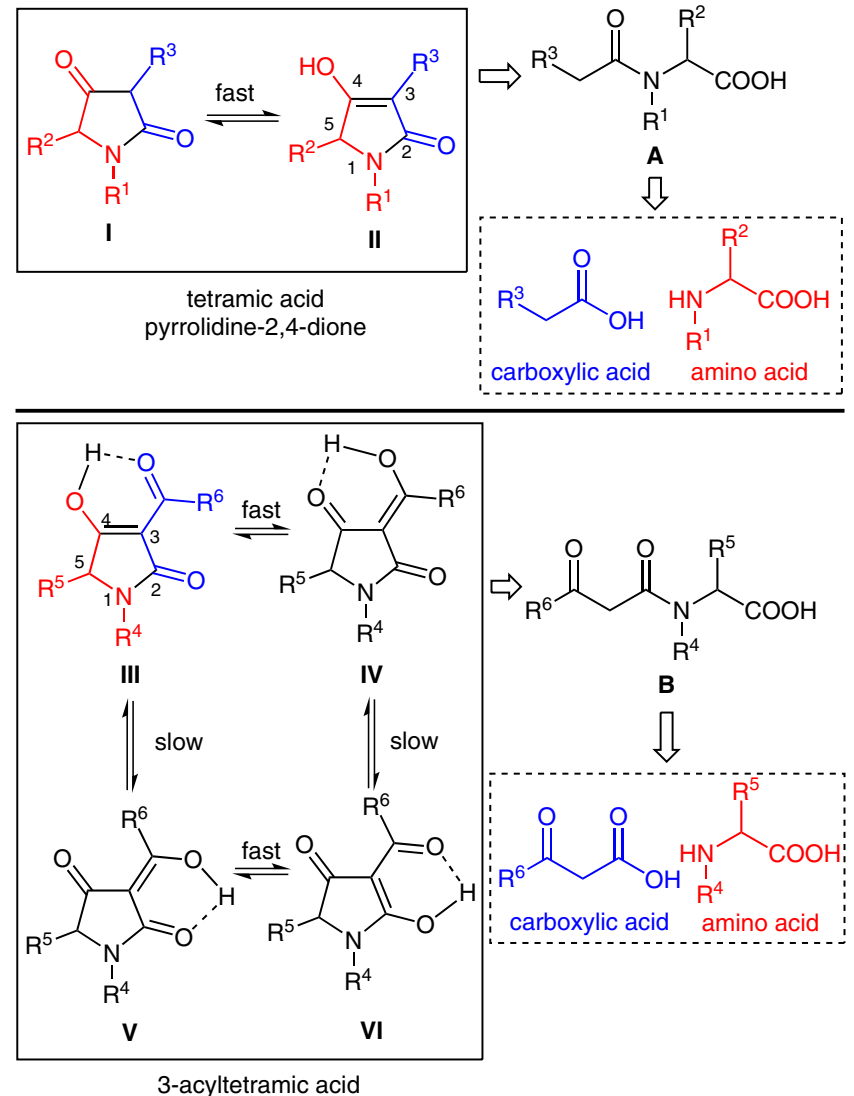

Scheme 1 Tetramic acids and 3-acyltetramic acids

mers, consisting of the two pairs of rapidly interconverting internal tautomers III/IV and V/VI. Biogenetically, the construction of tetramic acids and 3-acyltetramic acids might involve the formation of bond between the C-3 and C-4 atoms by an intramolecular Dieckmann cyclization of the corresponding peptide ( $\mathbf{A}$ and $\mathbf{B}$, respectively); this, in turn, might be derived from a carboxylic acid and an amino acid. Because of their unique structures and potential biological activities, naturally occurring tetramic acids have attracted a considerable degree of interest from organic chemists since the 1960 s. $^{6}$

(+)-Fusarisetin A (1; Figure 1), a new 3-acyltetramic acid, was isolated from the soil fungus Fusarium sp. FN080326 by Ahn and co-workers in $2011 .^{7}$ As a fungal metabolite, $(+)$-fusarisetin $\mathrm{A}$ is a potent inhibitor of acinar morphogenesis, cell migration, and cell invasion in MDA-MB231 cells. Investigations of cell growth and cell death in this breast-cancer cell line indicated that $(+)$-fusarisetin A does not exhibit significant cytotoxicity. ${ }^{7}$ These findings suggest that (+)-fusarisetin A might be a valuable anticancer agent, especially for the inhibition of cancer-cell metathesis. Besides its promising biological activity, (+)fusarisetin A (1) has some interesting structural features. It contains a pentacyclic ring system $(\mathrm{A}-\mathrm{B}-\mathrm{C}-\mathrm{D}-\mathrm{E})$ that includes a trans-decalin moiety (rings $\mathrm{A}$ and $\mathrm{B}$ ), a spiro moiety (rings $\mathrm{C}$ and $\mathrm{E}$ ), and a 3-acyltetramic acid moiety (ring E). It has ten stereocenters, including two all-carbon quaternary centers ( $\mathrm{C}-1$ and $\mathrm{C}-16)$ and a hetero-quaternary center $(\mathrm{C}-4)$. Ahn and co-workers confirmed the relative configuration of (+)-fusarisetin A by x-ray crystallographic analysis. The absolute stereochemistry of 1 was originally assigned by means of the exciton chirality circular dichroism method, but was subsequently revised by Li and co-workers as a result of their chemical synthesis. ${ }^{8 \mathrm{a}}$ The molecule has attracted considerable attention from the synthetic chemistry community since its discovery. To date, four groups, including ourselves, have accomplished total syntheses of fusarisetin $\mathrm{A}$. Li and coworkers achieved the first synthesis by using a palladiumcatalyzed oxygen-to-carbon allylic rearrangement to give

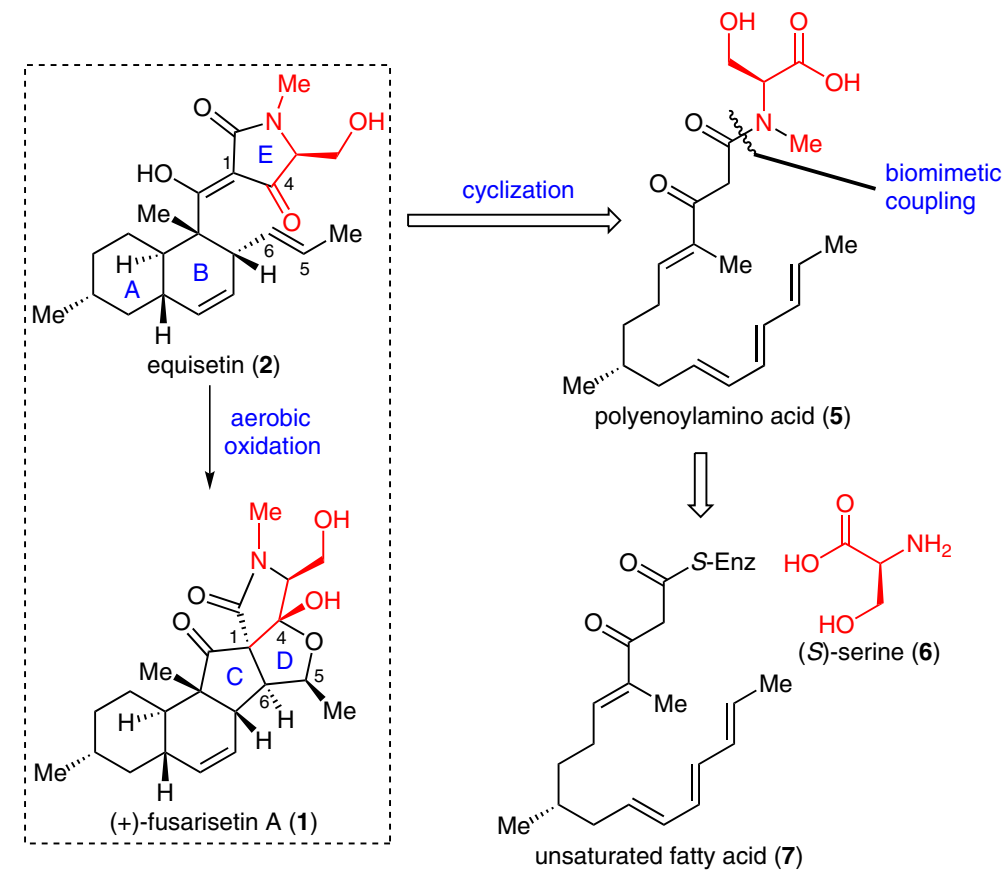

Scheme 2 Research plan based on a biosynthetic hypothesis 
the enantiomer (-)-1; as a result, they revised the absolute configuration of the molecule. ${ }^{8 a}$ Theodorakis and coworkers proposed a biosynthetic pathway from equisetin (2) and they accomplished a biomimetic synthesis of (-)1 by a one-pot radical cyclization/aminolysis approach. ${ }^{8 b, c}$ Recently, Yang and co-workers reported a synthesis of $(+)-1$ in which their intramolecular version of the PausonKhand reaction served as a key step. ${ }^{8 \mathrm{~d}}$

Our research group ${ }^{9}$ is interested in the synthesis of bioactive natural products with anticancer potential. We considered that (+)-fusarisetin A (1) would be a perfect target for the exploration of its laboratory synthesis and biological functions. During our retrosynthetic analysis, we conducted a careful literature search that showed that, in addition to (+)-fusarisetin $\mathrm{A}$, several other tetramic acids can be isolated from fungi. These biogenetically related 3acyltetramic acids include equisetin (2), ${ }^{10}$ trichosetin (3), ${ }^{11}$ and ophiosetin (4) ${ }^{12}$ (Figure 1). Equisetin (2) was isolated from the white mold Fusarium equiseti in 1974, and it shows strong antibiotic activity, HIV inhibitory activity, and selective cytotoxicity to mammalian cells. ${ }^{10}$ Surprisingly, we found that both (+)-fusarisetin A (1) and<smiles>CCOC(=O)/C(C)=C/CC[C@H](C)CC=O</smiles>

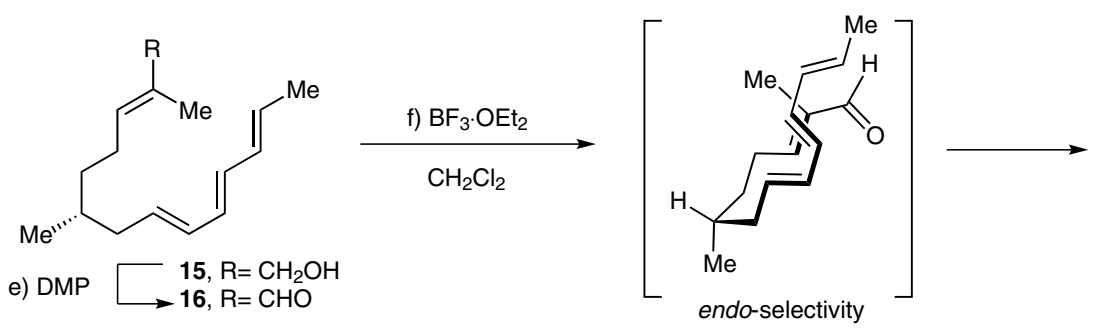

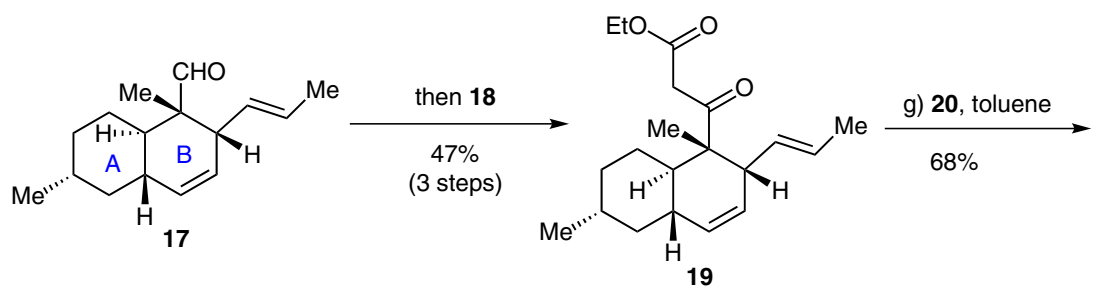

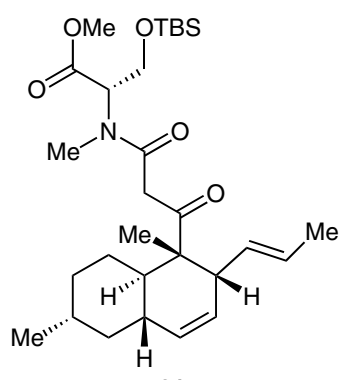

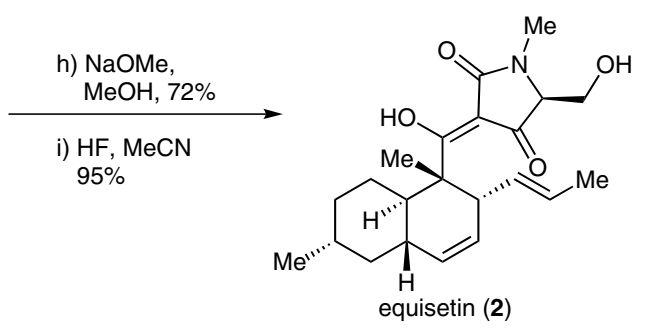<smiles>CCOC(=O)C(C)=[Pb]</smiles><smiles>C/C=C/C=C/CP(=O)(OCC)OCC</smiles><smiles>CCOC(=O)C=[W]</smiles><smiles>COC(=O)C(COC(C)(C)C)NN(C)C</smiles>

Scheme 3 First-generation synthesis of equisetin (2) 
equisetin (2) have similar natural sources and similar structural properties, including the basic skeletal rings A, $\mathrm{B}$, and $\mathrm{E}$, although they differ in their oxidation states. It is reasonable to assume that $(+)$-fusarisetin $\mathrm{A}$ and equisetin are biogenetically related and share a common biosynthetic pathway from the polyenoylamino acid 5 (Scheme 2 ). Acid 5 might be derived by coupling of naturally occurring $(S)$-serine (6) with the unsaturated fatty acid 7 . An intramolecular Diels-Alder reaction, ${ }^{13}$ followed by a Dieckmann cyclization, should transform acid $\mathbf{5}$ into equisetin (2). We surmised that the keto-enol tautomers of the 3 -acyltetramic acid moiety in equisetin should be readily oxidizable to give a radical that might be further transformed into (+)-fusarisetin A through aerobic oxidation, C1-C6 bond formation, and oxidation of C-5.

Guided by this biosynthetic analysis, we planned to synthesize equisetin (2) first and then to use it as a biogenetic precursor of (+)-fusarisetin A (1). We developed two syntheses of equisetin (2). Our first-generation synthesis was based on the pioneering studies of Danishefsky, ${ }^{14 a}$ Ley, ${ }^{14 \mathrm{~b}, \mathrm{c}}$ and Shishido ${ }^{14 \mathrm{~d}}$ and their respective co-workers. The chiral methyl group at C-12 of both 1 and 2 could be produced from $(+)$-citronellal $(\mathbf{8}) .{ }^{15}$ As shown in Scheme 3 , this approach is straightforward and efficient. By using classical chemical transformations, we were able to prepare aldehyde $\mathbf{1 6}$ from aldehyde 9 in five steps. The Lewis acid-promoted intramolecular Diels-Alder ${ }^{13}$ reaction stereospecifically transformed aldehyde $\mathbf{1 6}$ into the transdecalin 17 (the A and B rings) with endo-selectivity. ${ }^{14 a}$ This intramolecular Diels-Alder reaction and the subsequent Roskamp reaction could be mediated by the same Lewis acid (boron trifluoride etherate). We then installed the 3-acyltetramic acid moiety (E ring) of equisetin by using the aminolysis/Dieckmann cyclization sequence developed by Danishefsky. ${ }^{14 a}$ Simple deprotection of the tert-butyl(dimethyl)silyl group then gave equisetin (2) in $95 \%$ yield. This first-generation approach gave equisetin (2) in 11 steps from the commercially available $(+)$-citronellal (8). However, the efficiency of this approach did not satisfy us, so we planned to develop a biomimetic synthesis of $\mathbf{2}$, based on our biosynthetic hypothesis, starting from the polyenoylamino acid $\mathbf{5}$ and involving a cyclization sequence.

Our second-generation synthesis began with the preparation of polyenoylamino ester 27, the methyl ester of 5, which contains all the required parts of equisetin. Aldehyde 9 was efficiently converted into the unsaturated thio ester 25 by a sequence of two Horner-WadsworthEmmons reactions with phosphonates $22^{14 b, c}$ and 13, respectively (Scheme 4). Ester 27 was then prepared by coupling ester 25 with the $(S)$-serine derivative 26. The intramolecular Diels-Alder reaction of 27 smoothly gave the desired trans-decalin A and B rings of ester 29. The stereoselectivity of this reaction was substrate-controlled and involved the chair-like transition state $\mathbf{2 8}$. Finally, ester 29 was converted into equisetin (2) by Dieckmann cyclization under basic conditions. ${ }^{14 \mathrm{~b}, \mathrm{c}}$ We also induced the Dieckmann cyclization and then performed the intramo- lecular Diels-Alder reaction by using the polyenoylamino ester 27. Under chemical conditions, without the help of an enzyme, we obtained a mixture of four diastereomers. We therefore consider $\mathbf{2 9}$ to be involved in the biosynthesis of equisetin (2). In comparison with our first-generation synthesis, our second-generation approach gives equisetin (2) more efficiently in eight steps from (+)-citronellal (8), and is capable of being scaled up.
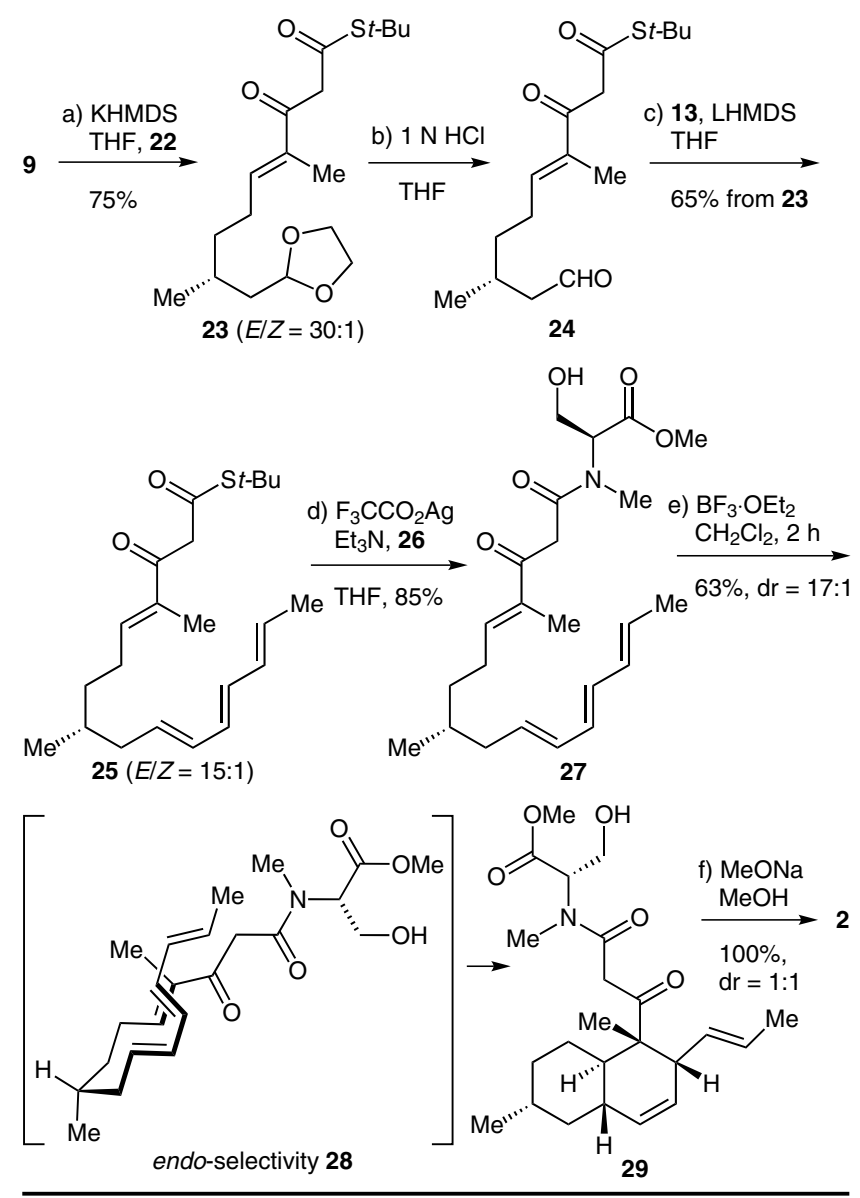<smiles>CCOP(=O)(OCC)C(C)C(=O)CC(=O)S(C)(C)C</smiles><smiles>CNC(CO)C(=O)OC</smiles>

Scheme 4 Second-generation synthesis of equisetin (2)

Having developed a scalable synthesis of equisetin (2), we began to investigate its bioconversion into (+)-fusarisetin A (1). On the basis of our proposed biogenetic relationship between $\mathbf{1}$ and $\mathbf{2}$, we believed that aerobic oxidation of 2 should give a key radical intermediate that should undergo an aerobic radical cascade process to form the $\mathrm{C} 1-$ $\mathrm{C} 6$ bond ( $\mathrm{C}$ ring) as well as oxidizing $\mathrm{C}-5$ to close the $\mathrm{D}$ ring through hemiketalization. We selected manganese(III) acetate ${ }^{16}$ as a single-electron oxidant to initiate the aerobic radical cascade, because this reagent is suitable for use with enolizable carbonyl compounds. ${ }^{16}$ The 3acyltetramic acid (E ring) moiety theoretically contains a tricarbonyl group that should be readily oxidized by man- 
ganese(III) acetate. Because the radical intermediate might also be trapped by oxygen to form endoperoxides or hydroperoxides under aerobic conditions, we planned to use the manganese(III)/dioxygen conditions ${ }^{17}$ for the oxidation of equisetin to effect simultaneous closure of the $\mathrm{C}$ ring and selective oxidation of the $\mathrm{C}-5$ atom.

We extensively surveyed the reaction conditions and were pleased to find that oxidation of equisetin (2) with a stoichiometric amount of manganese(III) acetate under air or oxygen $(1 \mathrm{~atm})$ in acetic acid at room temperature gave peroxyfusarisetin 32 and its C5-epimer 33 in $62 \%$ combined yield $(\mathrm{dr}=1.3: 1)$ (Scheme 5). The relative stereochemistry of $\mathbf{3 3}$ was unambiguously confirmed by X-ray crystallography. We believe that the reaction of manganese(III) acetate with the 3-acyltetramic acid moiety in equisetin gives the manganese enolate $\mathbf{3 0}$, which then directly cyclizes in a 5-exo-trig manner to form the $\mathrm{C} 1-\mathrm{C} 6$ bond ( $\mathrm{C}$ ring), giving the radical intermediate 31 . This radical intermediate is trapped by dioxygen and hemiketalizated to form the peroxy derivatives $\mathbf{3 2}$ and $\mathbf{3 3}$ under aerobic conditions. We surmised that the termination of this radical cascade involves a hydrogen abstraction from equisetin. If this were so, the reaction should be initiated by a catalytic amount of manganese(III) acetate. Indeed, we found that the reaction of equisetin with $10 \mathrm{~mol} \%$ of manganese(III) acetate gave the peroxides 32 and $\mathbf{3 3}$ in a yield $(60 \%, \mathrm{dr}=1.1: 1)$ that was comparable to that of the noncatalytic process. Note that Theodorakis and co-workers have reported that cerium(IV) ammonium nitrate is an effective oxidant for initiating this reaction. ${ }^{8 \mathrm{c}}$

To examine further the biosynthesis of (+)-fusarisetin A (1) from equisetin (2), especially the biooxidation of 2 to peroxyfusarisetin, we needed to consider the reaction as a natural process. By analogy to photosynthesis, ${ }^{18}$ the biosynthetic aerobic oxidation requires three components: an energy source, a means of energy transfer, and an oxidant.
Undoubtedly, solar energy (visible light) is the basic energy source, and its energy is normally transformed, by means of a photosensitizer, into chemical energy that can be stored. Dioxygen acts as a potent oxidant in nature. We therefore speculated that the desired biooxidation of equisetin to form (+)-fusarisetin A might be initiated by visible light and a photocatalyst in the presence of air or oxygen. With this idea in mind, we designed two distinct approaches test this hypothesis. The first approach involved an organometallic photocatalyst-promoted, aerobic, radical-cascade process, and the second involved a dye-type photocatalyst-mediated cyclization process involving singlet oxygen.

To test the first approach with an organometallic photocatalyst (Scheme 6), a solution of equisetin (2) in acetonitrile containing a catalytic amount of tris(bipyridyl)dichlororuthenium (35) ${ }^{19}$ and triethylamine (4.0 equiv) under air or oxygen was irradiated by sunlight or light from a household compact fluorescence lamp. The desired peroxyfusarisetin $\mathbf{3 2}$ and its C5-epi-isomer $\mathbf{3 3}$ were obtained $(\mathrm{dr}=2: 1)$ in $68 \%$ combined yield. These results suggest that the superoxide radical anion $\left(\mathrm{O}_{2}{ }^{-}\right),{ }^{20} \mathrm{a}$ reactive oxygen species ${ }^{21}$ plays a key role in this aerobic oxidation. The superoxide radical anion is generated by reduction of dioxygen by the ruthenium complex. We believe that the superoxide radical anion acts as an initiator for the reaction by abstracting hydrogen from equisetin (2) to give the key radical intermediate $\mathbf{3 4}$, which undergoes the same reaction as that which occurs under manganese(III)/dioxygen conditions, to give $\mathbf{3 2}$ and $\mathbf{3 3}$.

Next, we examined the use of a dye-type photocatalyst, and we found that the oxidative cyclization reaction of equisetin (2) with methylene blue (37) as the photocatalyst gave 32 and $\mathbf{3 3}(\mathrm{dr}=3: 1)$ in $70 \%$ yield (Scheme 6$)$. In contrast to the reaction catalyzed by the ruthenium complex, singlet oxygen $\left({ }^{l} \mathrm{O}_{2}\right),{ }^{22}$ another reactive oxygen species, is
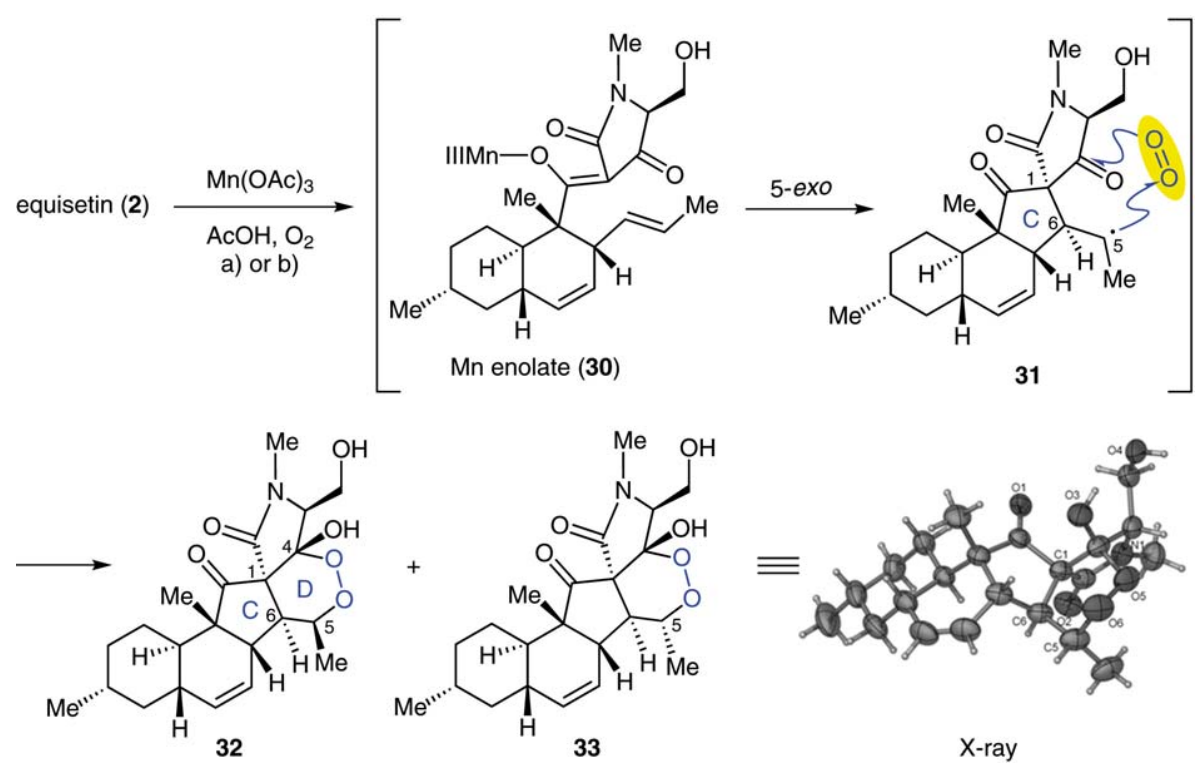

Scheme 5 Aerobic oxidation of equisetin to peroxyfusarisetin promoted by $\mathrm{Mn}(\mathrm{III}) / \mathrm{O}_{2}$. Reagents and conditions: $(\mathrm{a}) \mathrm{Mn}(\mathrm{OAc})_{3} \cdot 2 \mathrm{H}_{2} \mathrm{O}(2.0$ equiv), air or $\mathrm{O}_{2}(1 \mathrm{~atm}), 25^{\circ} \mathrm{C}, 1 \mathrm{~h}: 62 \%(\mathrm{dr}=1.3: 1$ at $\mathrm{C}-5)$; (b) $\mathrm{Mn}(\mathrm{OAc})_{3} \cdot 2 \mathrm{H}_{2} \mathrm{O}\left(0.1\right.$ equiv), air or $\mathrm{O}_{2}(1 \mathrm{~atm}), 25^{\circ} \mathrm{C}, 2 \mathrm{~h}: 60 \%(\mathrm{dr}=1.1: 1 \mathrm{at}$ C-5). 

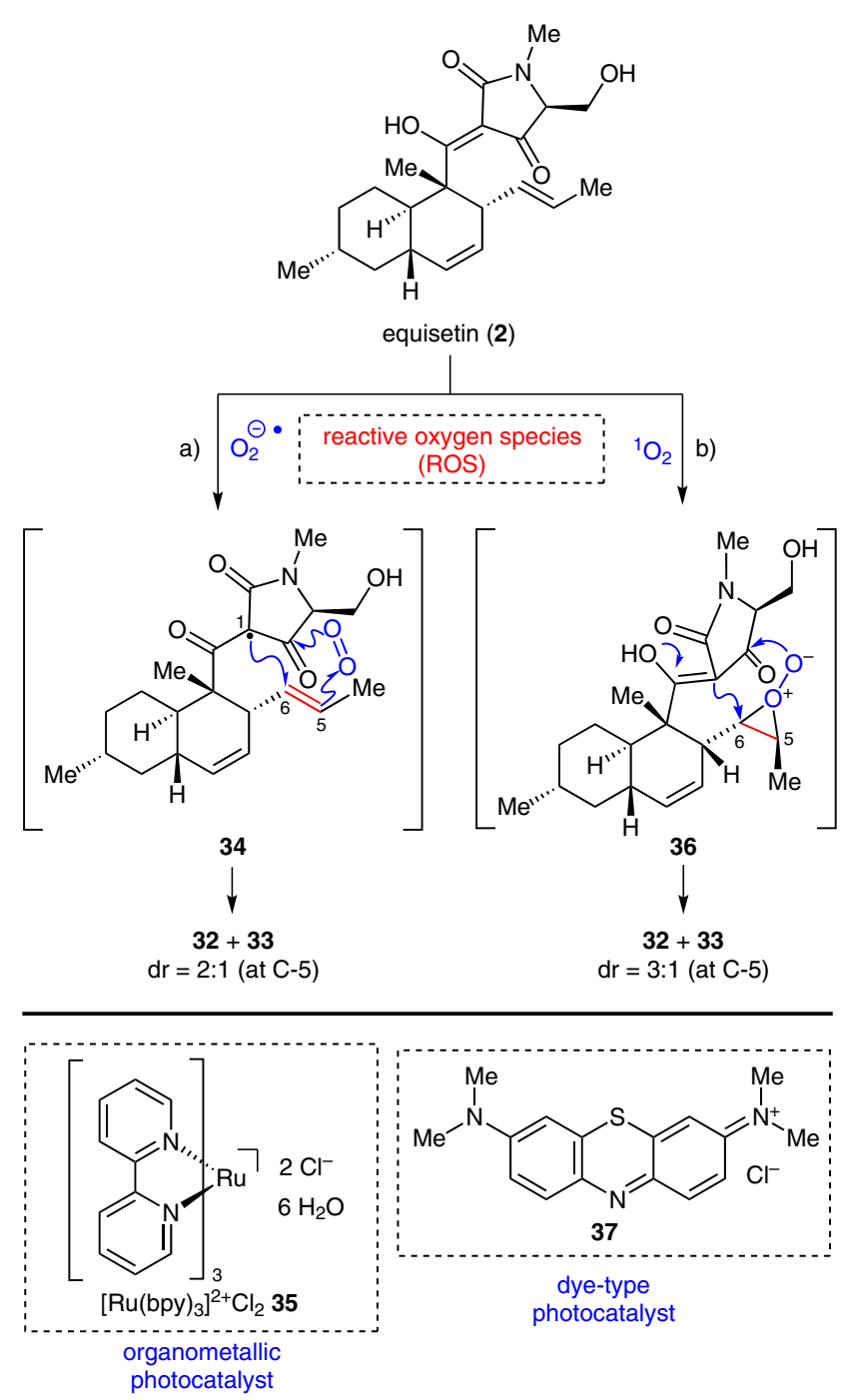

Scheme 6 Aerobic oxidation of equisetin to peroxyfusarisetin mediated by photochemically generated reactive oxygen species. Reagents and conditions: (a) $\mathrm{Ru}(\mathrm{bpy})_{3} \mathrm{Cl}_{2}(5 \mathrm{~mol} \%), \mathrm{Et}_{3} \mathrm{~N}$ (4.0 equiv), under air or $\mathrm{O}_{2}$, compact fluorescent lamp or sunlight, $23{ }^{\circ} \mathrm{C}, 4 \mathrm{~h}: 68 \%(\mathrm{dr}=2: 1$ at $\mathrm{C}-5$ ); (b) methylene blue (5 mol\%), $\mathrm{Et}_{3} \mathrm{~N}$ (4.0 equiv), under air or $\mathrm{O}_{2}$, compact fluorescent lamp or sunlight, $25^{\circ} \mathrm{C}, 4 \mathrm{~h}: 70 \%(\mathrm{dr}=3: 1$ at C-5).

generated in this reaction. The photoexcited methylene blue $37^{*}$ transfers light energy to activate the triplet oxygen to form singlet oxygen $\left({ }^{I} \mathrm{O}_{2}\right)$, which, in turn, reacts selectively with the $\mathrm{C} 5-\mathrm{C} 6$ double bond to form $\mathbf{3 6}$; this undergoes rearrangement to form $\mathbf{3 2}$ and $\mathbf{3 3}$. Both the organometallic and dye-type photocatalyzed reaction conditions are physiologically relevant, suggesting that a reactive oxygen species may be involved in the biosynthesis of (+)-fusarisetin A from equisetin.

To complete our synthesis of (+)-fusarisetin A (1), we needed to cleave the peroxide bonds reductively to form the tetrahydrofuran ring (D ring). Reduction of the mixture of $\mathbf{3 2}$ and $\mathbf{3 3}$ with zinc and acetic acid gave (+)-fusarisetin A (1) and 5-epi-1 ${ }^{8 \mathrm{a}}$ in $75 \%$ combined yield (Scheme 7). Biogenetically, the single-electron reduction would be performed by a phosphine- or sulfur-containing compound. Indeed, we found that this reduction could be induced by using trimethyl phosphite to give 1 and C5epi-1 in 75\% yield (Scheme 7). Theodorakis and co-workers have reported that thiourea is also an effective reagent for this reduction. ${ }^{8 c}$

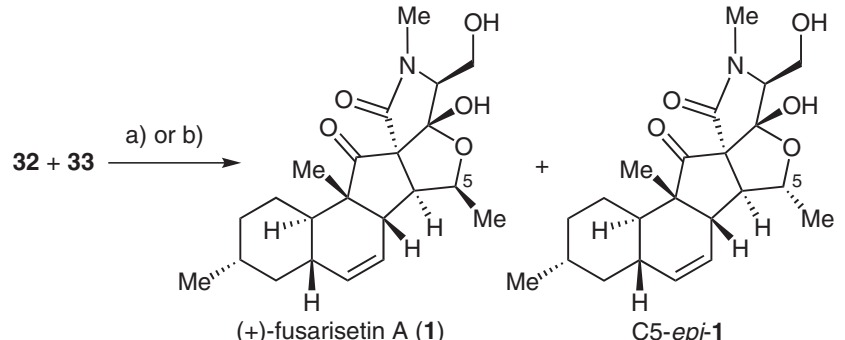

Scheme 7 Synthesis of (+)-fusarisetin A (1) by reduction of peroxyfusarisetin. Reagents and conditions: (a) $\mathrm{Zn}$ (30.0 equiv), $\mathrm{AcOH}$, $50{ }^{\circ} \mathrm{C}, 2 \mathrm{~h}: 75 \%$ (combined); (b) $\mathrm{P}(\mathrm{OMe})_{3}$, (40.0 equiv), $\mathrm{MeCN}$, $80{ }^{\circ} \mathrm{C}, 3$ h: $75 \%$ (combined).

We were intrigued by the possibility of performing the aerobic oxidation and the subsequent single-electron reduction as a one-pot operation to provide a more convenient approach for preparing (+)-fusarisetin A (1) and, indeed, we were pleased to find that direct addition of zinc to the dioxygen-trapping oxidative radical sequence gave 1 in $41 \%$ yield from equisetin (Scheme 8). This permits the efficient preparation of $\mathbf{1}$ on a large scale in ten steps starting from (+)-citronellal (8).

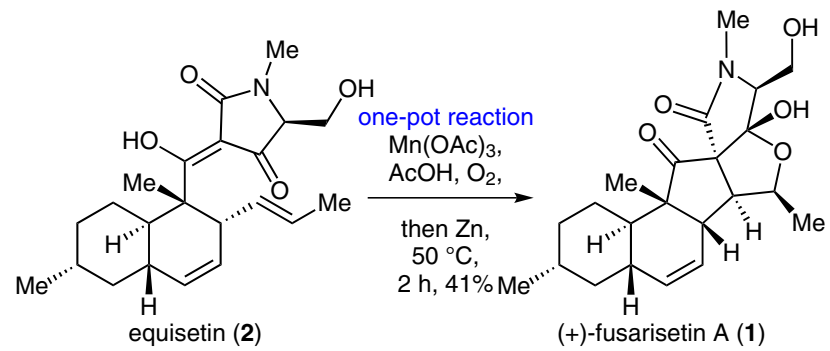

Scheme 8 Preparation of (+)-fusarisetin A (1) from equisetin (2) by a one-pot reaction

In summary, we have described our recent studies on syntheses of naturally occurring tetramic acids. An efficient total synthesis of $(+)$-fusarisetin A (1), a promising anticancer agent, was achieved by a biomimetic approach, starting from equisetin (2). Our research suggests that both $\mathbf{1}$ and $\mathbf{2}$ are derived from polyenoylamino acid. The biosynthesis of (+)-fusarisetin A (1) might involve an aerobic oxidation of equisetin (2), which could be mediated by metal oxidants or by photochemically produced reactive oxygen species. We are optimistic that this approach could provide biogenetically related natural products efficiently. We have already prepared a variety of derivatives of $\mathbf{1}$ by using a similar strategy. This will facilitate studies on structure-activity relationships and further studies on medicinal chemistry. 


\section{Acknowledgment}

Financial support was provided by the NSFC (21102045 and 21272076), the Shanghai Pujiang Program (11PJ1402800), RFDP (No. 20110076120022) of Higher Education of China, and the Program for Professor of Special Appointment (Eastern Scholar) at Shanghai institutions of higher learning (No. 1220000144). We also thank Prof. Xiaoli Zhao for performing the X-ray analysis.

\section{References}

(1) Newman, D. J. J. Nat. Prod. 2012, 75, 311.

(2) Nicolaou, K. C.; Montagnon, T. Molecules That Changed the World: A Brief History of the Art and Science of Synthesis and Its Impact on Society; Wiley: Weinheim, 2008.

(3) (a) Hendrickson, J. B. J. Am. Chem. Soc. 1975, 97, 5784. (b) Gaich, T.; Baran, P. S. J. Org. Chem. 2010, 75, 4657.

(4) (a) Trost, B. M.; Salzmann, T. N. J. Am. Chem. Soc. 1973, 95, 6840. (b) Trost, B. M. Science 1983, 219, 245.

(c) Shenvi, R. A.; O'Malley, D. P.; Baran, P. S. Acc. Chem. Res. 2009, 42, 530.

(5) For atom-economic synthesis, see: (a) Trost, B. M. Science 1991, 254, 1471. (b) Trost, B. M. Angew. Chem. Int. Ed. Engl. 1995, 34, 259. For step-economic synthesis, see: (c) Wender, P. A.; Croatt, M. P.; Witulski, B. Tetrahedron 2006, 62, 7505. (d) Wender, P. A.; Verma, V. A.; Paxton, T. J.; Pillow, T. H. Acc. Chem. Res. 2008, 41, 40. (e) Wender, P. A.; Miller, B. L. Nature 2009, 460, 197. For redoxeconomic synthesis, see: (f) Richter, J. M.; Ishihara, Y.; Masuda, T.; Whitefield, B. W.; Llamas, T.; Pohjakallio, A.; Baran, P. S. J. Am. Chem. Soc. 2008, 130, 17938. (g) Burns, N. Z.; Baran, P. S.; Hoffmann, R. W. Angew. Chem. Int. Ed. 2009, 48, 2854.

(6) For a review on tetramic acid type natural products, see: Royles, B. J. L. Chem. Rev. 1995, 95, 1981.

(7) Jang, J.-H.; Asami, Y.; Jang, J.-P.; Kim, S.-O.; Moon, D. O.; Shin, K.-S.; Hashizume, D.; Muroi, M.; Saito, T.; Oh, H.; Kim, B. Y.; Osada, H.; Ahn, J. S. J. Am. Chem. Soc. 2011, $133,6865$.

(8) For total syntheses of fusarisetin A, see: (a) Deng, J.; Zhu, B.; Lu, Z.; Yu, H.; Li, A. J. Am. Chem. Soc. 2012, 134, 920. (b) Xu, J.; Caro-Diaz, E. J. E.; Trzoss, L.; Theodorakis, E. A. J. Am. Chem. Soc. 2012, 134, 5072. (c) Xu, J.; Caro-Diaz, E. J. E.; Lacoske, M. H.; Hung, C.-I.; Jamora, C.; Theodorakis, E. A. Chem. Sci. 2012, 3, 3378. (d) Huang, J.; Fang, L.; Long, R.; Shi, L.-L.; Shen, H.-J.; Li, C.-C.; Yang, Z. Org. Lett. 2013, 15, 4018.

(9) (a) Yin, J.; Wang, C.; Kong, L. L.; Cai, S. J.; Gao, S. H. Angew. Chem. Int. Ed. 2012, 51, 7786. (b) Yin, J.; Kong, L. L.; Wang, C.; Shi, Y. B.; Cai, S. J.; Gao, S. H. Chem. Eur. J. 2013, 19, 13040. For a review on progress in the synthesis of fusarisetin A, see: (c) Yin, J.; Kong, L. L.; Gao, S. H. Youji Hиахие 2013, 33, 259.
(10) For the isolation of equisetin, see: (a) Burmeister, H. R.; Bennett, G. A.; Vesonder, R. F.; Hesseltine, C. W. Antimicrob. Agents Chemother. 1974, 5, 634. (b) Phillips, N. J.; Goodwin, J. T.; Fraiman, A.; Cole, R. J.; Lynn, D. G. J. Am. Chem. Soc. 1989, 111, 8223.

(11) Marfori, E. C.; Kajiyama, S.; Fukusaki, E.; Kobayashi, A. Z. Naturforsch., C 2002, 57, 465.

(12) Putri, S. P.; Kinoshita, H.; Ihara, F.; Igarashi, Y.; Nihira, T. J. Antibiot. 2010, 63, 195.

(13) For reviews on the Diels-Alder reaction in total synthesis, see: (a) Craig, D. Chem. Soc. Rev. 1987, 16, 187. (b) Nicolaou, K. C.; Snyder, S. A.; Montagnon, T.; Vassilikogiannakis, G. Angew. Chem. Int. Ed. 2002, 41, 1668. For a review on intramolecular Diels-Alder reactions in total synthesis, see: (c) Takao, K.; Munakata, R.; Tadano, K. Chem. Rev. 2005, 105, 4779.

(14) For total syntheses of equisetin, see: (a) Turos, E.; Audia, J. E.; Danishefsky, S. J. J. Am. Chem. Soc. 1989, 111, 8231. (b) Burke, L. T.; Dixon, D. J.; Ley, S. V.; Rodríguez, F. Org. Lett. 2000, 2, 3611. (c) Burke, L. T.; Dixon, D. J.; Ley, S. V.; Rodríguez, F. Org. Biomol. Chem. 2005, 3, 274.

(d) Kumiko, Y. K.; Shindo, M.; Shishido, K. Tetrahedron Lett. 2001, 42, 2517.

(15) Nishikawa, Y.; Kitajima, M.; Kogure, N.; Takayama, H. Tetrahedron 2009, 65, 1608.

(16) For reviews on $\mathrm{Mn}(\mathrm{III})-$ promoted radical reactions, see: (a) Snider, B. B. Chem. Rev. 1996, 96, 339. (b) Melikyan, G. G. Org. React. (N. Y.) 1997, 49, 427. For a review on the mechanisms of $\mathrm{Mn}(\mathrm{OAc})_{3}$-based radical reactions, see: (c) Snider, B. B. Tetrahedron 2009, 65, 10738

(17) For a review on $\mathrm{Mn}(\mathrm{III})$-based peroxidation, see: Nishino, $\mathrm{H}$. Top. Heterocycl. Chem. 2006, 6, 39.

(18) Gust, D.; Moore, T. A. Science 1989, 244, 35.

(19) (a) Cano-Yelo, H.; Deronzier, A. J. Chem. Soc., Perkin Trans. 2 1984, 1093. (b) MacMillan, D. W. C. Nature 2008, 455,304

(20) (a) Zen, J.-M.; Liou, S.-L.; Kumar, A. S.; Hsia, M.-S Angew. Chem. Int. Ed. 2003, 42, 577. (b) Zhang, M.; Chen, C.-C.; Ma, W.-H.; Zhao, J.-C. Angew. Chem. Int. Ed. 2008, 47, 9730. (c) Zou, Y. Q. L.; Lu, Q.; Fu, L.; Chang, N. J.; Rong, J.; Chen, J. R.; Xiao, W. J. Angew. Chem. Int. Ed. 2011, 50, 7171. (d) Xuan, J.; Cheng, Y.; An, J.; Lu, L. Q.; Zhang, X. X.; Xiao, W. J. Chem. Commun. 2011, 47, 8337. (e) Zou, Y. Q.; Chen, J. R.; Liu, X. P.; Lu, L. Q.; Davis, R. L.; Jørgensen, K. A.; Xiao, W. J. Angew. Chem. Int. Ed. 2012, 51, 784. (f) Su, Y.; Zhang, L.; Jiao, N. Org. Lett. 2011, 13,2168 .

(21) (a) Wipf, P.; Graham, T. H.; Xiao, J. Pure Appl. Chem. 2007, 79, 753. (b) Dickinson, B. C.; Chang, C. J. Nat. Chem. Biol. 2011, 7, 504

(22) For reviews on singlet oxygen chemistry, see: (a) Kearns, D. R. Chem. Rev. 1971, 71, 395. (b) Frimer, A. A. Chem. Rev. 1979, 79, 359. (c) George, M. V.; Bhat, V. Chem. Rev. 1979, 79, 447. (d) DeRosa, M. C.; Crutchley, R. J. Coord. Chem. Rev. 2002, 233-234, 351. 